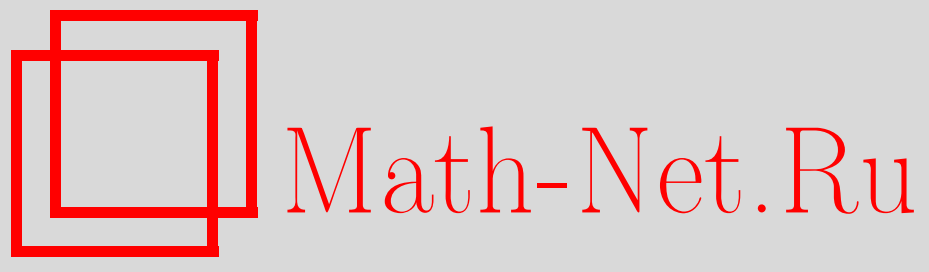

В. Л. Попов, Алгебраические конусы, Матем. заметки, 2009, том 86, выпуск 6, 947-949

DOI: https://doi.org/10.4213/mzm8529

Использование Общероссийского математического портала Math-Net.Ru подразумевает, что вы прочитали и согласны с пользовательским соглашением http: //www . mathnet.ru/rus/agreement

Параметры загрузки:

IP: 3.85 .7 .115

26 апреля 2023 г., 15:15:07

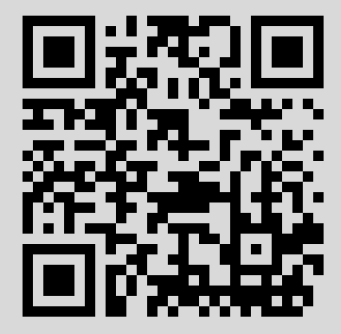




\section{Алгебраические конусы}

\section{В. Л. Попов}

В этой заметке дан ответ на вопрос К. Аджамагбо, поставленный в письме к автору от 9 июля 2009 г.

Все рассматриваемые ниже алгебраические многообразия определены над алгебраически замкнутым полем $k$.

Неприводимое алгебраическое многообразие $X$ называется конусом, если $X$ аффинно и его координатная алгебра $k[X]$ допускает связную $\mathbb{N}$-градуировку:

$$
k[X]=\bigoplus_{d \in \mathbb{N}} k[X]_{d}, \quad k[X]_{0}=k .
$$

Пусть $\mathbf{M}_{\mathrm{m}}$ - мультипликативный алгебраический моноид, подлежащим многообразием которого является аффинная прямая $\mathbf{A}^{1}$, а умножение $\mu: \mathbf{A}^{1} \times \mathbf{A}^{1} \rightarrow \mathbf{A}^{1}$ определено умножением в $k$, т.е. $\mu^{*}(T)=T \otimes T$, где $T$ - стандартная координатная функция на $\mathbf{A}^{1}$. Группой единиц в $\mathbf{M}_{\mathrm{m}}$ является $\mathbf{M}_{\mathrm{m}} \backslash\{0\}=\mathbf{G}_{\mathrm{m}}$. Обозначим

$$
\chi_{d}: \mathbf{G}_{\mathrm{m}} \rightarrow \mathbf{G}_{\mathrm{m}}, \quad t \mapsto t^{d},
$$

Мы говорим, что $\mathbf{M}_{\mathrm{m}}$ действует на многообразии $Y$, если задан такой морфизм $\alpha: \mathbf{M}_{\mathrm{m}} \times Y \rightarrow Y$, что $\alpha(g, \alpha(h, y))=\alpha(g h, y)$ и $\alpha(1, y)=y$ для любых $g, h, \in \mathbf{M}_{\mathrm{m}}, y \in Y$. Мы используем запись $g(y):=\alpha(g, y)$. Ограничение $\alpha$ на $\mathbf{G}_{\mathrm{m}} \times Y$ является обычным действием группы $\mathbf{G}_{\mathrm{m}}$ на $Y$. Множество $\mathbf{M}_{\mathrm{m}}(y):=\left\{g(y) \mid g \in \mathbf{M}_{\mathrm{m}}\right\}$ называется $\mathbf{M}_{m}$-орбитой точки $y$ (предупреждение: различные $\mathbf{M}_{m}$-орбиты могут иметь непустое пересечение). Если $\mathbf{M}_{\mathrm{m}}(y)=y$, то $y$ называется неподвижной точкой действия.

Теорема. Пусть $X$ - неприводимое алгебраическое многообразие. Рассмотрим следующие свойства:

(i) $X$ является конусом;

(ii) существует действие $\mathbf{M}_{\mathrm{m}}$ на Х с единственной неподвижной точкой;

(iii) существует действие $\mathbf{G}_{\mathrm{m}}$ на $X$ с неподвиюной точкой, которая является единственной замкнутой орбитой.

Тогда (i) $\Rightarrow$ (ii) $\Rightarrow$ (iii) и, если $X$ нормально, (iii) $\Rightarrow$ (i).

ДоказатеЛЬСтво. Мы можем считать, что $\operatorname{dim} X>0$.

(i) $\Rightarrow$ (ii) Пусть $X$ является конусом. Рассмотрим градуировку (1). Тогда формула $t \cdot f:=t^{d} f$, где $t \in \mathbf{G}_{m}, f \in k[X]_{d}$, определяет действие $\mathbf{G}_{m}$ на $k[X]$ автоморфизмами $k$-алгебры. В свою очередь, оно определяет (алгебраическое) действие $\mathbf{G}_{m}$ на $X$. Ввиду связности градуировки (1) мы имеем

$$
k[X]^{\mathbf{G}_{\mathrm{m}}}=k .
$$

Поскольку $k[X]^{\mathbf{G}_{\mathrm{m}}}$ разделяет замкнутые $\mathbf{G}_{\mathrm{m}}$-орбиты [1; следствие A1.3], из (3) и [2; следствие I.1.8] следует, что существует единственная такая орбита $O$. Координатная алгебра $k[O]$ многообразия $O$ не содержит $\mathbf{G}_{\mathrm{m}}$-стабильных собственных идеалов, поскольку множество нулей такого идеала было бы собственным $\mathbf{G}_{\mathrm{m}}$-стабильным подмножеством в $O$. Значит, поскольку из замкнутости $O$ следует сюръективность отображения

Работа выполнена при поддержке Российского фонда фундаментальных исследований (грант № 08-01-00095), программы "Ведущие научные школы" (грант № 1987.2008.1) и программы "Современные проблемы теоретической математики” отделения математики РАН.

(C) В. Л. Попов, 2009 
$k[X] \rightarrow k[O],\left.f \mapsto f\right|_{O}$, мы получаем, что $\mathbf{G}_{\mathrm{m}}$-стабильный идеал $\bigoplus_{d>0} k[X]_{d}$ обращается на $O$ в нуль. Следовательно, $O$ является точкой.

Ввиду теоремы вложения [3; теорема 1.5$]$ мы можем считать, что $X$ является замкнутым $\mathbf{G}_{\mathrm{m}}$-стабильным подмножеством конечномерного $\mathbf{G}_{\mathrm{m}}$-модуля $V$ и $X$ не содержится ни в каком его собственном подмодуле. Но множество нулей всех линейных функций на $V$, обращающихся в нуль на $X$, является подмодулем, содержащим $X$. Следовательно, мы имеем вложение $\mathbf{G}_{\mathrm{m}}$-модулей $V^{*} \hookrightarrow k[X],\left.\ell \mapsto \ell\right|_{X}$. Поскольку веса $V$ обратны весам $V^{*}$, отсюда и из $(3)$ следует, что нулевой вектор $0_{V}$ является единственной $\mathbf{G}_{\mathrm{m}}$-неподвижной точкой в $V$; в частности, $O=0_{V}$. Ввиду (1) отсюда также следует, что для любого $\mathbf{G}_{\mathrm{m}}$-веса $\chi_{d}$ модуля $V^{*}$ мы имеем $d \in \mathbb{N}$. Значит, ввиду (2) существуют числа $d_{1}, \ldots, d_{n} \in \mathbb{N}$ и базис $e_{1}, \ldots, e_{n}$ в $V$ такие, что $t\left(e_{i}\right)=t^{d_{i}} e_{i}$ для любых $t \in \mathbf{G}_{\mathrm{m}}$ и $i$. Это показывает, что, положив $0(v)=0_{V}$ для любого $v \in V$, мы получаем продолжение действия $\mathbf{G}_{m}$ на $V$. Поскольку $\mathbf{M}_{\mathrm{m}}$ является замыканием $\mathbf{G}_{\mathrm{m}}$, а $X$ замкнуто и $\mathbf{G}_{\mathrm{m}}$-стабильно, то $X$ также и $\mathbf{M}_{\mathrm{m}}$-стабильно. По той же причине $0_{V}$ является неподвижной точкой для $\mathbf{M}_{\mathrm{m}}$. Поскольку $V^{\mathbf{G}_{\mathrm{m}}}=\left\{0_{V}\right\}$, других неподвижных точек нет.

(ii) $\Rightarrow$ (iii) Предположим, что $\mathbf{M}_{m}$ действует на неприводимом многообразии $X$ с единственной неподвижной точкой $x$. Ограничим это действие на $\mathbf{G}_{m}$. Возьмем какую-либо точку $y \in X$. Если $\mathbf{G}_{m}$-орбита $\mathbf{G}_{m}(y)$ замкнута в $X$, то $\mathbf{G}_{m}(y)=\mathbf{M}_{m}(y)$, поскольку $\mathbf{G}_{m}$ плотно в $\mathbf{M}_{m}$.

Мы утверждаем, что тогда $\mathbf{G}_{m}(y)$ является точкой, а потому $\mathbf{G}_{m}(y)=x$ ввиду единственности $x$. В самом деле, допустим, что это не так, т.е. $\operatorname{dim} \mathbf{G}_{m}(y)=1$. Орбитное отображение $\varphi_{y}: \mathbf{M}_{\mathrm{m}} \rightarrow \mathbf{M}_{m}(y), g \mapsto g(y)$, является сюръективным морфизмом одномерных гладких многообразий, его слои конечны и для любой точки $z \in \mathbf{M}_{\mathrm{m}}(y), z \neq \varphi_{y}(0)$, число точек в слое $\varphi_{y}^{-1}(z)$ равно порядку $\mathbf{G}_{\mathrm{m}}$-стабилизатора точки $y$ (обозначим его через $s$ ), тогда как число точек в слое $\varphi_{y}^{-1}\left(\varphi_{y}(0)\right)$ равно $s+1$. Согласно [4; раздел 2 , следствие 2] это невозможно; противоречие. Таким образом, $x$ - единственная замкнутая $\mathbf{G}_{m}$-орбита.

(iii) $\Rightarrow$ (i) Предположим теперь, что $\mathbf{G}_{\mathrm{m}}$ действует на $X$ с единственной неподвижной точкой $x$ и других замкнутых орбит нет. Тогда для каждой точки $y \in X, y \neq x$, замыкание $\overline{\mathbf{G}_{m}(y)}$ орбиты $\mathbf{G}_{m}(y)$ одномерно и

$$
x \in \overline{\mathbf{G}_{m}(y)} .
$$

Предположим далее, что $X$ нормально. Тогда по теореме Сумихиро [5; следствие 2 леммы 8] существует $\mathbf{G}_{\mathrm{m}}$-стабильная аффинная открытая окрестность $U$ точки $x$. Мы утверждаем, что $X=U$. В самом деле, если это не так, то $X \backslash U$ является непустым $\mathbf{G}_{\mathrm{m}}$-стабильным подмножеством в $X$ и (4) невозможно для $y \in X \backslash U$; противоречие.

Таким образом, $X=U$ и, значит, $X$ аффинно.

Поскольку функции из $k[X]^{\mathbf{G}_{\mathrm{m}}}$ постоянны на $\mathbf{G}_{\mathrm{m}}$-орбитах, из (4) следует, что $f(y)=$ $f(x)$ для любых $f \in k[X]^{\mathbf{G}_{\mathrm{m}}}$ и $y \in X ;$ следовательно, выполнено (3).

Пусть $k[X]_{d}$ обозначает $\chi_{d}$-изотипную компоненту $\mathbf{G}_{\mathrm{m}}$-модуля $k[V]$. Тогда

$$
k[X]=\bigoplus_{d \in \mathbf{Z}} k[X]_{d}
$$

является $\mathbb{Z}$-градуировкой $k$-алгебры $k[X]$. Ввиду (3) она связна, т.е. $k[X]_{0}=k$.

Мы утверждаем, что не существует таких целых чисел $d_{1}>0$ и $d_{2}<0$, что $k[X]_{d_{i}} \neq 0$ при $i=1,2$. В самом деле, предположим, что это не так. Тогда существует такая точка $y \in X$, что $k[X]_{d_{i}}, i=1,2$, не лежит в ядре отображения $k[X] \rightarrow k\left[\overline{\mathbf{G}_{m}(y)}\right],\left.f \mapsto f\right|_{\overline{\mathbf{G}_{m}(y)}}$. Значит, $\chi_{d_{i}}$-изотипная компонента $\mathbf{G}_{m}$-модуля $k\left[\overline{\mathbf{G}_{m}(y)}\right]$ является ненулевой для каждого $i=1,2$. Отсюда следует, что существует такое целое число $d \neq 0$, что $\mathbf{G}_{m}$-стабильный максимальный идеал $\left\{f \in k\left[\overline{\mathbf{G}_{m}(y)}\right] \mid f(x)=0\right\}$ в $k\left[\overline{\mathbf{G}_{m}(y)}\right]$ имеет ненулевые изотипные компоненты весов $\chi_{d}$ и $\chi_{d}^{-1}$. Пусть $p$ и $q$ - ненулевые элементы соответственно первой 
и второй из них. Тогда функция $p q$ постоянна на $\mathbf{G}_{m}(y)$, а следовательно, и на $\overline{\mathbf{G}_{m}(y)}$. Поскольку $p q(x)=0$, это означает, что $p q=0$ вопреки неприводимости $\overline{\mathbf{G}_{m}(y)}$; противоречие.

Таким образом, $k[X]_{d}=0$ в (5) либо для всех отрицательных, либо для всех положительных $d$. Заменяя, если необходимо, действие $\mathbf{G}_{m}$ на $X$ на $g \cdot y:=g^{-1}(y)$, мы можем считать, что реализуется первая возможность, т.е. выполнено (1). Это завершает доказательство.

ЗАмечАниЕ. Следующий пример показывает, что в общем случае без предположения о нормальности $X$ импликации (iii) $\Rightarrow$ (i) и (ii) $\Rightarrow$ (i) не имеют места.

ПримеР. Пусть $X$ - образ морфизма

$$
\begin{gathered}
\nu: \mathbf{P}^{1} \rightarrow \mathbf{P}^{2}, \quad\left(a_{0}: a_{1}\right) \mapsto\left(p^{3}: q^{2} t-p^{3}: q^{3}-q p^{2}\right), \\
p=a_{1}-a_{0}, \quad q=a_{1}+a_{0} .
\end{gathered}
$$

Многообразие $X$ является проективной плоской кубикой с обычной двойной точкой $O=$ $(1: 0: 0)$, а $\mathbf{P}^{1} \rightarrow X, x \mapsto \nu(x),-$ ее нормализацией. Формула $\alpha\left(t,\left(a_{0}: a_{1}\right)\right)=\left(a_{0}: t a_{1}\right)$ определяет действие $\mathbf{G}_{\mathrm{m}}$ на $\mathbf{P}^{1}$, которое спускается на $X$ с помощью $\nu$, см. [6]. Для этого действия $O$ является единственной неподвижной точкой, а $X \backslash O$ - орбитой. Оно продолжается до действия $\mathbf{M}_{\mathrm{m}}$, при котором $0(x)=O$ для любой точки $x \in X$.

\section{СПИСОК ЦИТИРОВАННОЙ ЛИТЕРАТУРЫ}

[1] D. Mumford, J. Fogarty, Geometric Invariant Theory, Ergeb. Math. Grenzgeb., 34, Springer-Verlag, Berlin, 1982. [2] A. Borel, Linear Algebraic Groups, Grad. Texts in Math., 126, Springer-Verlag, New York, 1991. [3] Э. Б. Винберг, В. Л. Попов, Алгебраическая геометрия - 4, Итоги науки и техн. Сер. Соврем. пробл. мат. Фундам. направления, 55, ВИНИТИ, М., 1989, 137-309. [4] А. Grothendieck, Classification de groupes de Lie algébriques, Séminaire Claude Chevalley, 1956-1958, 1, Secrétariat mathématique, Paris, 1958, Exposé no. 5, 19 pp. [5] H. Sumihiro, J. Math. Kyoto Univ., 14 (1974), 1-28. [6] В. Л. Попов, Матем. заметки, 23:2 (1978), 183-195.

\section{В. Л. Попов}

Поступило

Математический институт им. В. А. Стеклова РАН

01.09.2009

E-mail: popovvl@orc.ru 